\title{
Terapia da Insuficiência Cardíaca Avançada Ajustada por Objetivos Hemodinâmicos Obtidos pela Monitorização Invasiva
}

Advanced Heart Failure Therapy Adapted to Hemodynamic Objectives Acquired from Invasive Hemodynamic Monitoring

Gustavo Luiz Gouvêa de Almeida Júnior, Roberto Esporcatte, Fernando Oswaldo Dias Rangel, Ricardo Mourilhe Rocha, Gustavo de Miranda Gouvêa e Silva, Bernardo Rangel Tura, José Kezen Camilo Jorge, Luiz Eduardo Fonseca Drumond, Francisco Manes Albanesi Filho Hospital Pró-Cardíaco, Universidade do Estado do Rio de Janeiro e Casa de Saúde São José - Rio de Janeiro, RJ

\section{Objetivo}

Avaliar o tratamento da insuficiência cardíaca (IC) avançada em relação à redução das pressões de enchimento ventricular, com a utilização de doses maiores de vasodilatadores, através da monitorização hemodinâmica invasiva.

\section{Métodos}

Foram estudados 19 pacientes com IC avançada, nos quais foi instalado o cateter de Swan-Ganz para guiar a administração de diurético intravenoso (IV) e nitroprussiato de sódio, com o objetivo de se reduzir de forma significativa as pressões de enchimento ventricular. Depois de alcançado esse objetivo ou 48 horas, foram introduzidas drogas orais até serem retirados os fármacos venosos, mantendo o benefício hemodinâmico.

\section{Resultados}

Dos 19 pacientes estudados, 16 (84\%) eram do sexo masculino. A idade média foi de $66 \pm 11,4$ anos; a fração de ejeção média foi de $26 \pm 6,3 \% ; 2$ pacientes $(10,5 \%)$ apresentavam classe funcional (CF) III e 17 (89,5\%), CF IV. Houve queda da pressão de oclusão da artéria pulmonar de $23 \pm 11,50 \mathrm{mmHg}$ para $16 \pm 4,05 \mathrm{mmHg}(p=0,008)$, do índice de resistência vascular sistêmica de $3.023 \pm$ $1.153,71$ dynes $/ \mathrm{s} / \mathrm{cm}^{-5} / \mathrm{m}^{2}$ para $1.834 \pm 719,34$ dynes $/ \mathrm{s} /$ $\mathrm{cm}^{-5} / \mathrm{m}^{2}(\mathrm{p}=0,0001)$ e aumento do índice cardíaco de 2,1 $\pm 0,56 \mathrm{l} / \mathrm{min} / \mathrm{m}^{2}$ para $2,8 \pm 0,73 \mathrm{l} / \mathrm{min} / \mathrm{m}^{2}(p=0,0003)$. Um subgrupo com hipovolemia foi identificado.

\section{Conclusão}

Foi possível reduzir as pressões de enchimento ventricular para valores significativamente menores, obtendo melhora significativa do índice cardíaco, do índice de resistência vascular sistêmica e da pressão média da artéria pulmonar, utilizando-se doses significativamente maiores de vasodilatadores.

\section{Palavras-chave}

monitorização hemodinâmica invasiva, insuficiência cardíaca, terapia ajustada, tratamento

\section{ОвJective}

To assess advanced heart failure (HF) treatment in relation to reduction of ventricular filling pressures, with the use of greater doses of vasodilators, through invasive hemodynamic monitoring.

\section{Methods}

Nineteen advanced HF patients were studied, in whom Swan-Ganz catheter was inserted to direct administration of diuretic intravenously (IV) and sodium nitroprusside, with the aim of significantly reduce ventricular filling pressures. After achieving such objective or 48 hours, oral drugs were introduced until venous medicines were removed, keeping hemodynamic benefit.

\section{RESULTS}

From 19 patients studied, 16 (84\%) were of male sex. The average age was $66 \pm 11.4$ years old; average ejection fraction was $26 \pm 6.3 \%$; 2 patients (10.5\%) showed functional class (FC) III and 17 (89.5\%), FC IV. There was a decrease of pulmonary artery occlusion pressure from $23 \pm 11.50 \mathrm{mmHg}$ to $16 \pm$ $4.05 \mathrm{mmHg}(p=0.008)$, of systemic vascular resistance index from 3,023 $\pm 1,153.71 \mathrm{dynes} / \mathrm{s} / \mathrm{cm}^{-5} / \mathrm{m}^{2}$ to $1,834 \pm 719.34$ dynes $/ \mathrm{s} / \mathrm{cm}^{-5} / \mathrm{m}^{2}(p=0.0001)$ and an increase of cardiac index from $2.1 \pm 0.56 \mathrm{l} / \mathrm{min} / \mathrm{m}^{2}$ to $2.8 \pm 0.73 \mathrm{l} / \mathrm{min} / \mathrm{m}^{2}$ ( $p=0.0003$ ). A subgroup with hypovolemia was identified.

\section{Conclusion}

It was possible to reduce ventricular filling pressures to significantly lower values, obtaining a significant improvement of cardiac index, systemic vascular resistance index and pulmonary artery mean pressure, by using significantly higher doses of vasodilators.

\section{KEY WORDS}

invasive hemodynamic monitoring, heart failure, adjusted therapy, treatment 
A avaliação clínica de pacientes com insuficiência cardíaca (IC), com base apenas no exame físico, tem se mostrado inadequada, tanto para o diagnóstico diferencial de função sistólica preservada ou não ${ }^{1,2}$, quanto para estimar a condição hemodinâmica desses pacientes ${ }^{3}$. Naqueles com disfunção ventricular importante, essa dificuldade de ajuste hemodinâmico pode resultar em tratamento subótimo e perpetuação da condição de hipervolemia, baixo débito e piora funcional. A mortalidade dessa população com IC avançada continua elevada apesar do surgimento de novas estratégias terapêuticas. Em época anterior ao emprego dos beta-bloqueadores, a mortalidade desse grupo era estimada em 36\% em um ano, como no estudo CONSENSUS ${ }^{4}$, e em 37\% em 6 meses, no estudo FIRST $^{5}$. Já na época dos beta-bloqueadores, a mortalidade anual, observada no estudo COPERNICUS ${ }^{6}$, foi de $11,4 \%$ nos pacientes tratados com carvedilol e de $18,5 \%$, nos tratados com placebo.

O transplante cardíaco é a melhor terapêutica disponível para esta população. No entanto, encontra aplicação limitada devido à reduzida oferta de doadores. Terapêuticas alternativas vêm sendo cada vez mais estudadas, visando à melhoria da qualidade e quantidade de vida dos pacientes. Exemplos desses tratamentos são a cirurgia de D'Or, a terapia da ressincronização ventricular ${ }^{7,8}$ e o implante de células-tronco no miocárdio ${ }^{9}$, entre outros. A utilização da monitorização hemodinâmica invasiva (MHI), com cateter de Swan-Ganz para guiar a terapêutica farmacológica da IC avançada, vem sendo utilizada nos últimos anos com resultados promissores ${ }^{10-}$ 12. Com ela, é possível reduzir, na maioria dos casos, as pressões de enchimento ventricular de forma significativa, separar subgrupos de maior gravidade que não respondem ao tratamento, melhorar sintomas e retirar pacientes da fila de transplante ${ }^{13}$.

Este estudo teve por objetivo utilizar a estratégia de tratamento de pacientes com IC avançada, baseado nos dados obtidos através da MHI e observar se é possível reduzir significativamente as pressões de enchimento ventricular, utilizando doses elevadas de vasodilatadores e diuréticos. Foram avaliadas, ainda, as conseqüências dessa estratégia nos parâmetros laboratoriais de função renal e eletrolítica.

\section{MÉtodos}

Foi estudada de forma prospectiva, no período de 01/ 02/99 a 15/05/02, uma amostra de conveniência, constituída de 19 pacientes com IC avançada. Todos foram internados em ambiente de terapia intensiva, onde foi realizado o protocolo. Os dados clínicos e funcionais da população estudada encontram-se na tabela I.

Quanto à etiologia, 10 pacientes eram isquêmicos, dois idiopáticos, um hipertensivo, cinco valvares e um alcoólico.

Os critérios de inclusão utilizados foram: disfunção

\begin{tabular}{|c|c|}
\hline Dados clínicos e funcionais & População estudada ( $n=19)$ \\
\hline Idade (anos) & $66 \pm 11,4$ \\
\hline Sexo masculino & $16(84 \%)$ \\
\hline Classe funcional III & $2(10,5 \%)$ \\
\hline Classe funcional IV & $17(89,5 \%)$ \\
\hline Fração de ejeção (\%) & $26 \pm 6,3$ \\
\hline
\end{tabular}

(FE) ao ecocardiograma $<30 \%$, pelo método de Teichholz ou análise subjetiva, uma vez que na inclusão alguns pacientes poderiam estar em uso de aminas vasoativas, superestimando o valor da FE, e classe funcional III ou IV da NYHA (New York Heart Association).

Os critérios de exclusão utilizados foram: condições não-cardíacas que tivessem expectativa de óbito em menos de um ano, tais como neoplasias, síndrome da imunodeficiência adquirida etc., e infarto agudo do miocárdio ou angina instável nos últimos três meses.

Através de acesso venoso profundo (veia subclávia ou jugular), puncionado por técnica de Seldinger, foi inserido cateter fluxo dirigido (Swan-Ganz), modelo débito cardíaco e $\mathrm{SVO}_{2}$ (saturação venosa mista de oxigênio) contínuos da Baxter ${ }^{\circledR}$ e, através de acesso arterial (artéria radial, femoral ou pediosa), puncionada por técnica de Seldinger, foi instalado cateter para a monitorização da pressão arterial média.

Após a colocação dos cateteres, cada paciente permaneceu pelo menos 1 hora em repouso, antes das medidas hemodinâmicas. As medidas foram feitas com os pacientes em decúbito dorsal e cabeceira do leito até $30^{\circ}$. A torneira de abertura para a atmosfera, ligada ao transdutor, foi nivelada a um ponto imaginário, formado pela linha axilar média na altura do $4^{\circ}$ espaço intercostal. $\mathrm{O}$ sistema foi então zerado e a $\mathrm{SVO}_{2}$ calibrada. Somente após esses cuidados, foram realizadas as medidas hemodinâmicas, que incluíram os seguintes parâmetros: pressão arterial sistólica (PAS), diastólica e média; pressão sistólica, diastólica e média da artéria pulmonar; pressão de oclusão da artéria pulmonar (POAP) e pressão de átrio direito (PAD). Foi obtido, ainda, o índice cardíaco e calculada a resistência vascular sistêmica (IRVS). A medicação oral foi suspensa após a colocação dos cateteres, sendo utilizados, então, nitroprussiato de sódio e diurético de alça intravenoso (IV) de acordo com os valores obtidos através da monitorização. Objetivavamse os seguintes valores hemodinâmicos: POAP $\leq 15$ $\mathrm{mmHg}$, IRVS $<2.100$ dynes $/ \mathrm{s} / \mathrm{cm}^{-5} / \mathrm{m}^{2}, \mathrm{PAD}<8 \mathrm{mmHg}$, PAS $>80 \mathrm{mmHg}$. Quando alcançada uma POAP $\leq 20$ $\mathrm{mmHg}$, os vasodilatadores orais eram iniciados, como mostra o fluxograma (fig.1), porém, sempre buscando como objetivo final valores $\leq 15 \mathrm{mmHg}$.

O fluxograma de realização do protocolo foi desenvolvido pelos autores, de acordo com a estratégia prevista no estudo. 


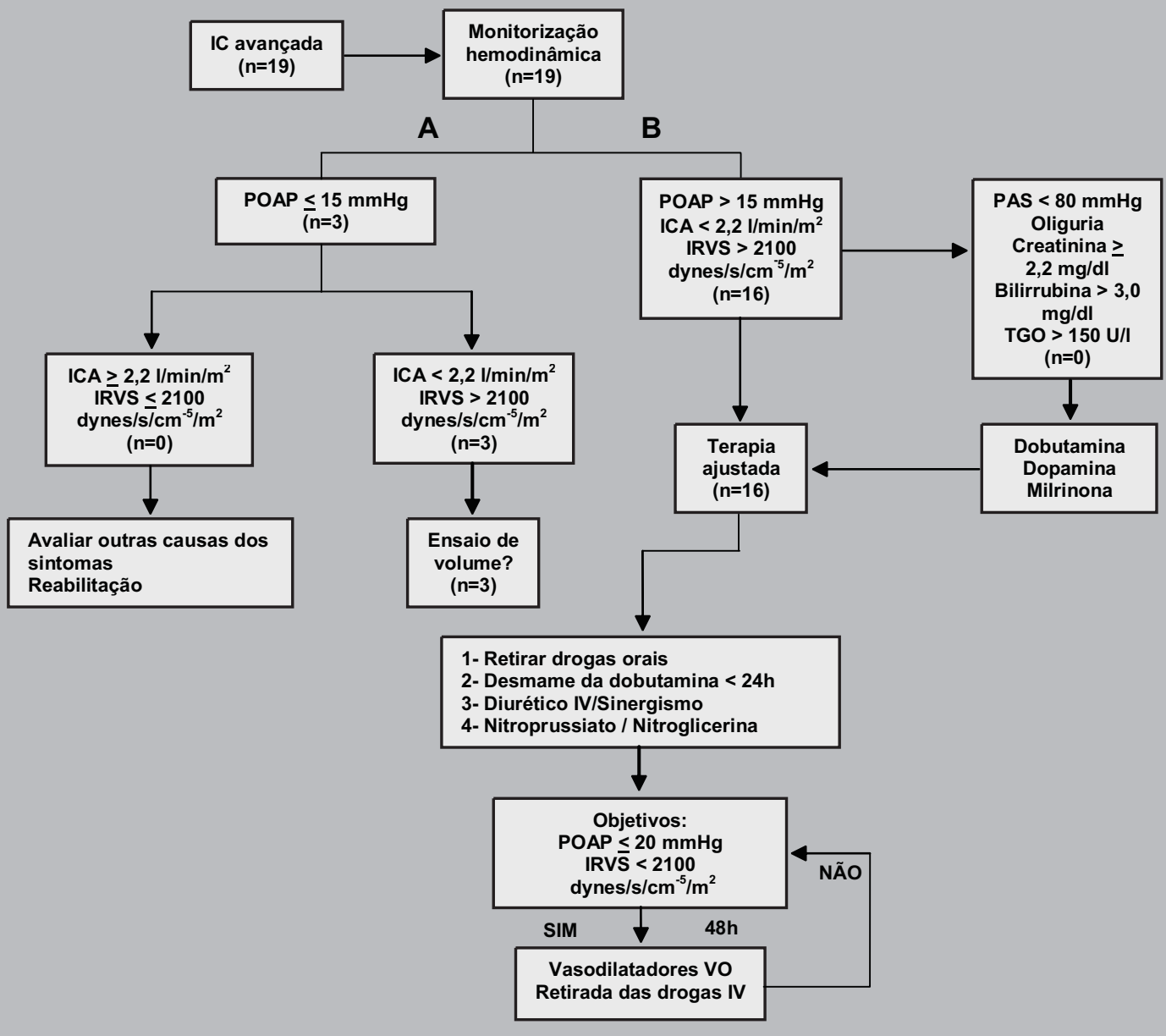

Fig. 1 - Fluxograma do protocolo realizado.

Os pacientes com valores hemodinâmicos iniciais típicos para a realização do protocolo (caminho B do fluxograma) foram tratados, fundamentalmente, com nitroprussiato de sódio IV e diurético de alça IV, sem qualquer fármaco oral até que os objetivos hemodinâmicos fossem alcançados ou após 48 horas do início do protocolo. 0 passo seguinte foi o início de captopril na dose de $6,25 \mathrm{mg}$ ou $12,5 \mathrm{mg}$ de acordo com a pressão arterial; a cada período de 6 horas a sua dose foi dobrada até um máximo de $50 \mathrm{mg}$ de 6/6h.

Progressivamente, o nitroprussiato de sódio foi retirado. Caso as medidas hemodinâmicas alcançadas com as medicações venosas não se mantivessem com a dose máxima de captopril, era associado o mononitrato de isossorbida, iniciando-se com dose de $20 \mathrm{mg}$ e dobrando a dose a cada 6 horas até o máximo de 80 mg de 6/6h. Nos pacientes que não alcançaram pressões de enchimento e IRVS ótimas no regime tolerado de captopril + nitrato, foi adicionada a hidralazina, visando maximizar a vasodilatação e a resposta hemodinâmica. A medicação foi iniciada com $12,5 \mathrm{mg}$ ou $25 \mathrm{mg}$ e dobrada a cada 6 horas até a dose máxima de 100 mg de 6/6h. A dose de diurético foi individualizada, de acordo com a resposta hemodinâmica, variando de 40 mg a 200mg a cada 6, 8 ou 12 horas. Naqueles pacientes resistentes a altas doses de diurético de alça, foi associado tiazídico e/ou espironolactona, com o intuito de bloqueio seqüencial do néfron. A utilização de inotrópicos foi sistematicamente evitada, em função dos seus efeitos deletérios e ausência de benefícios clínicos nos ensaios científicos ${ }^{14-16}$. Porém, naqueles pacientes com resposta inicial insatisfatória ao esquema apresentado, fundamentalmente quando foram observados pressão arterial sistólica (PAS) $<80 \mathrm{mmHg}$, débito urinário muito reduzido e/ou sinais de hipoperfusão orgânica refletido por aumento de transaminases, escórias ou bilirrubinas, foi permitido o uso da dobutamina, sendo descontinuado o seu uso assim que possível (objetivando não ultrapassar 24 horas).

Após a retirada das medicações venosas, com a manutenção do resultado hemodinâmico à custa dos vasodilatadores orais e diuréticos, a terapêutica adjuvante da IC foi prescrita (antiarrítmicos, anticoagulantes, digital, ácido acetilsalicílico, espironolactona etc). A medida dos 
dados hemodinâmicos foi feita várias vezes ao dia e, obrigatoriamente, antes e 1 hora após a administração das medicações orais ou ajuste dos fármacos venosos.

No mesmo dia da colocação dos cateteres e início do protocolo, foram realizados os seguintes exames: hemograma, glicose, uréia, creatinina, sódio, potássio, magnésio, coagulograma, hepatograma e marcadores de necrose miocárdica (CK total, CK-mb, Troponina I e CK-massa).

Os valores dos dados hemodinâmicos foram expressos como mediana \pm desvio padrão. Foi utilizado o teste de Wilcoxon para comparar os dados hemodinâmicos. Para avaliar a relação entre os fatores hemodinâmicos, foi utilizada a análise de correlação múltipla através do rho de Spearman; para corrigir o efeito da inter-relação e/ou interdependência dos parâmetros, foi utilizada uma análise de componentes principais. Todas as análises foram realizadas considerando uma significância de 95\%.

Foi obtido consentimento dos pacientes para a sua inclusão no protocolo.

\section{Resultados}

Após a obtenção das medidas hemodinâmicas iniciais, foi possível observar a presença de dois grupos distintos de pacientes: aqueles que apresentaram parâmetros iniciais de hipovolemia e aqueles com padrão típico de IC descompensada, com congestão sistêmica e resistência vascular periférica elevada. Apesar de os critérios de inclusão contemplarem apenas pacientes com grave disfunção ventricular e em classe funcional III e IV (NYHA), um subgrupo de 3 pacientes se apresentou, inicialmente, com valores compatíveis com hipovolemia, refletida por baixas pressões de enchimento ventricular, reduzido índice cardíaco e elevada resistência vascular sistêmica. Estes pacientes mostravam dificuldade em progredir com as doses de vasodilatadores por hipotensão arterial e persistência em CF IV, além de estarem em uso de diuréticos, o que mantinha a condição de hipovolemia. Depois de identificada esta condição, foi realizada reposição volêmica até que se atingissem as pressões de enchimento propostas. Isso proporcionou um aumento no índice cardíaco de 52,3\% (2,1 $\pm 0,28 \mathrm{l} / \mathrm{min} / \mathrm{m}^{2}$ para $3,2 \pm 0,25 \mathrm{l} / \mathrm{min} / \mathrm{m}^{2}$ ), queda no IRVS em 38,1\% (3.076 $\pm 116,14 \mathrm{dynes} / \mathrm{s} / \mathrm{cm}^{-5} / \mathrm{m}^{2}$ para $1.902 \pm 227,60 \mathrm{dynes} /$ $\mathrm{s} / \mathrm{cm}^{-5} / \mathrm{m}^{2}$ ) e adequação das pressões de enchimento ventricular. Com isso, foi possível progredir com os vasodilatadores até as doses propostas nos pacientes desse subgrupo. Os parâmetros hemodinâmicos de cada paciente deste grupo se encontram na tabela II.

Os pacientes do subgrupo de hipervolemia (tab. III) também mostraram exuberantes benefícios com a estratégia utilizada. Houve, após o término do protocolo, significativa melhora do índice cardíaco $(42,7 \%$ de aumento, passando a mediana de 1,85 $\pm 0,59 \mathrm{l} / \mathrm{min} / \mathrm{m}^{2}$ para 2,64 $\pm 0,75 \mathrm{l} / \mathrm{min} / \mathrm{m}^{2}$ ), redução do IRVS em $40 \%$ (2.972 $\pm 1.259,3 \mathrm{dynes} / \mathrm{s} / \mathrm{cm}^{-5} / \mathrm{m}^{2}$ para $1.784 \pm 781,3$ dynes $\left./ \mathrm{s} / \mathrm{cm}^{-5} / \mathrm{m}^{2}\right)$, queda da POAP em 37,7\% (26,5 \pm $8,5 \mathrm{mmHg}$ para $16,5 \pm 4 \mathrm{mmHg}$ ) e da PVC em 38,4\% $(13 \pm 4,5 \mathrm{mmHg}$ para $8 \pm 4,3 \mathrm{mmHg})$. Vale ressaltar que três pacientes $(9,17,18)$ apresentavam inicialmente POAP entre $20 \mathrm{mmHg}$ e $15 \mathrm{mmHg}$ e foram considerados como hipervolêmicos e descompensados. A mesma estratégia para aqueles com POAP > $20 \mathrm{mmHg}$ foi utilizada, o que resultou em aumento expressivo do índice cardíaco nos três e queda da resistência vascular periférica nos dois últimos.

Em relação ao grupo populacional, a análise da adequação das variáveis hemodinâmicas, antes e após o protocolo, indica que, à exceção da pressão venosa central, todas as outras medidas mostraram significativas melhoras. A tabela IV mostra essas comparações e respectivos valores de $p$.

As doses de vasodilatadores conseguidas ao final do protocolo foram significativamente maiores que as utilizadas antes, com o tratamento ambulatorial. A dose média de captopril utilizada antes e após o ajuste hemodinâmico foi, respectivamente, de 43,3 \pm 43,1 mg e 159,8 $\pm 61,2 \mathrm{mg}(\mathrm{p}=0,0009)$.

Cinco pacientes já estavam em uso de dobutamina quando entraram no protocolo. Em três deles, a infusão foi suspensa com menos de 24 horas, em um, com menos de 48 horas e no último, com menos de 36 horas, todos sem prejuízo hemodinâmico. Em nenhum caso foi necessário início de aminas vasoativas após a MHI.

Tabela II - Parâmetros hemodinâmicos iniciais e finais dos pacientes que apresentavam hipovolemia inicial

\begin{tabular}{|c|c|c|c|c|c|c|c|c|c|c|c|c|}
\hline $\mathrm{Pac}$ & $\begin{array}{l}\text { PAMi } \\
\mathrm{mmHg}\end{array}$ & $\begin{array}{l}\text { PAMf } \\
\mathrm{mmHg}\end{array}$ & $\begin{array}{l}\text { POAPi } \\
\mathrm{mmHg}\end{array}$ & $\begin{array}{l}\text { POAPf } \\
\mathrm{mmHg}\end{array}$ & $\begin{array}{l}\text { PVCi } \\
\mathrm{mmHg}\end{array}$ & $\begin{array}{l}\text { PVCf } \\
\mathrm{mmHg}\end{array}$ & $\begin{array}{c}\text { IRVSi } \\
\text { dynes/s/cm-5/m2 }\end{array}$ & $\begin{array}{c}\text { IRVSf } \\
\text { dynes/s/cm-5/m2 }\end{array}$ & $\begin{array}{c}\mathrm{ICi} \\
\mathrm{L} / \mathrm{min} / \mathrm{m} 2\end{array}$ & $\begin{array}{c}\text { ICf } \\
\mathrm{L} / \mathrm{min} / \mathrm{m} 2\end{array}$ & $\begin{array}{l}\text { PAPMi } \\
\mathrm{mmHg}\end{array}$ & $\begin{array}{l}\text { PAPMf } \\
\mathrm{mmHg}\end{array}$ \\
\hline 19 & 86 & 80 & 11 & 16 & 4 & 11 & 3.076 & 1.623 & 2,1 & 3,4 & 22 & 27 \\
\hline 10 & 77 & 75 & 4 & 10 & 1 & 6 & 2.894 & 1.902 & 2,1 & 2,9 & 17 & 19 \\
\hline 11 & 106 & 90 & 2 & 14 & 0 & 7 & 3.110 & 2.074 & 2,6 & 3,2 & 15 & 24 \\
\hline Mediana/ & 86 & 80 & 4 & 14 & 1 & 7 & 3.076 & 1.902 & 2,1 & 3,2 & 17 & 24 \\
\hline desvio- & \pm & \pm & \pm & \pm & \pm & \pm & \pm & \pm & \pm & \pm & \pm & \pm \\
\hline padrão & 14,84 & 7,63 & 4,72 & 3,05 & 2,08 & 2,64 & 116,14 & 227,60 & 0,28 & 0,25 & 3,60 & 4,04 \\
\hline
\end{tabular}

Pac- paciente; PAMi- pressão arterial média inicial; PAMf- pressão arterial média final; POAPi- Pressão de oclusão de artéria pulmonar inicial; POAPf-pressão de oclusão de artéria pulmonar final; PVCi-pressão venosa central inicial; PVCf- pressão venosa central final; IRVSi= Índice de resistência vascular sistêmica inicial; IRVSf-índice de resistência vascular sistêmica final; ICi- índice cardíaco inicial; ICf-índice cardíaco final; PAPMi- pressão arterial pulmonar média inicial; PAPMf- pressão arterial pulmonar média final. 
Tabela III - Parâmetros hemodinâmicos iniciais e finais dos pacientes que apresentavam hipervolemia inicial

\begin{tabular}{|c|c|c|c|c|c|c|c|c|c|c|c|c|}
\hline $\mathrm{Pac}$ & $\begin{array}{l}\text { PAMi } \\
\mathrm{mmHg}\end{array}$ & $\begin{array}{l}\text { PAMf } \\
\mathrm{mmHg}\end{array}$ & $\begin{array}{l}\text { POAPi } \\
\mathrm{mmHg}\end{array}$ & $\begin{array}{l}\text { POAPf } \\
\mathrm{mmHg}\end{array}$ & $\begin{array}{l}\mathrm{PVCi} \\
\mathrm{mmHg}\end{array}$ & $\begin{array}{l}\text { PVCf } \\
\mathrm{mmHg}\end{array}$ & $\begin{array}{c}\text { IRVSi } \\
\text { dynes/s/cm-5/m2 }\end{array}$ & $\begin{array}{c}\text { IRVSf } \\
\text { dynes/s/cm-5/m2 }\end{array}$ & $\begin{array}{c}\mathrm{ICi} \\
\mathrm{L} / \mathrm{min} / \mathrm{m} 2\end{array}$ & $\begin{array}{c}\text { ICf } \\
\mathrm{L} / \mathrm{min} / \mathrm{m} 2\end{array}$ & $\begin{array}{l}\text { PAPMi } \\
\mathrm{mmHg}\end{array}$ & $\begin{array}{l}\text { PAPMf } \\
\mathrm{mmHg}\end{array}$ \\
\hline 1 & 80 & 83 & 38 & 15 & 15 & 6 & 4.286 & 2.729 & 1,7 & 2,5 & 58 & 31 \\
\hline 2 & 98 & 61 & 36 & 11 & 12 & 2 & 3.851 & 2.477 & 1,8 & 1,9 & 47 & 21 \\
\hline 3 & 75 & 65 & 35 & 15 & 11 & 4 & 2.807 & 1.734 & 1,8 & 2,8 & 39 & 16 \\
\hline 4 & 86 & 57 & 30 & 9 & 13 & 5 & 4.233 & 1.608 & 1,3 & 2,5 & 34 & 22 \\
\hline 5 & 84 & 68 & 24 & 22 & 6 & 10 & 3.489 & 1.834 & 1,7 & 2,5 & 36 & 37 \\
\hline 6 & 97 & 57 & 23 & 17 & 13 & 14 & 5.504 & 2.567 & 1,2 & 1,3 & 43 & 29 \\
\hline 7 & 67 & 65 & 23 & 21 & 21 & 15 & 1.932 & 1.355 & 1,9 & 2,9 & 32 & 30 \\
\hline 8 & 71 & 52 & 22 & 12 & 13 & 8 & 2.108 & 1.599 & 2,2 & 2,2 & 28 & 26 \\
\hline 9 & 103 & 114 & 18 & 16 & 12 & 8 & 2.719 & 2.723 & 2,2 & 2,9 & 43 & 28 \\
\hline 12 & 96 & 73 & 34 & 19 & 19 & 14 & 5.726 & 3.692 & 1,0 & 1,2 & 45 & 33 \\
\hline 13 & 86 & 59 & 29 & 24 & 17 & 12 & 2.072 & 960 & 2,6 & 3,9 & 46 & 33 \\
\hline 14 & 82 & 66 & 35 & 18 & 9 & 4 & 3.736 & 2.833 & 1,5 & 1,7 & 38 & 26 \\
\hline 15 & 100 & 90 & 45 & 18 & 17 & 8 & 3.023 & 2.358 & 2,2 & 2,7 & 46 & 29 \\
\hline 16 & 65 & 57 & 20 & 20 & 14 & 15 & 1.133 & 1.018 & 3,5 & 3,3 & 40 & 45 \\
\hline 17 & 90 & 50 & 16 & 15 & 6 & 7 & 2.921 & 1.074 & 2,3 & 3,2 & 38 & 37 \\
\hline 18 & 84 & 79 & 19 & 14 & 6 & 5 & 2.667 & 1.627 & 2,3 & 3,6 & 26 & 19 \\
\hline Mediana/ & 85 & 65 & 26,5 & 16,5 & 13 & 8 & 2.972 & 1.784 & 1,85 & 2,64 & 39,5 & 29 \\
\hline desvio- & \pm & \pm & \pm & \pm & \pm & \pm & \pm & \pm & \pm & \pm & \pm & \pm \\
\hline padrão & 11,7 & 16,4 & 8,5 & 4,0 & 4,5 & 4,3 & $1.259,3$ & 781,3 & 0,59 & 0,75 & 7,97 & 7,40 \\
\hline
\end{tabular}

Apesar da intensa diurese obtida e da redução importante da pressão de enchimento ventricular, não se observou piora das escórias nitrogenadas, nem indução de distúrbio eletrolítico significativo, uma vez que houve intensa vigilância laboratorial (tab.V).

\section{Discussão}

O tratamento farmacológico dos pacientes com IC, baseado apenas no exame físico, tem sido a regra no manejo desses pacientes ao longo dos anos. Ultimamente, vem sendo questionado o paradigma de que esses pacientes, em especial, aqueles com grave disfunção ventricular, necessitam de pressões de enchimento elevadas para manter débito cardíaco e função renal adequados.

Neste estudo, pode-se observar que pacientes com IC avançada apresentam-se desajustados do ponto de vista hemodinâmico, apesar de estarem em uso prévio de digital, diuréticos e vasodilatadores. Ressalte-se a presença de três pacientes apresentando hipovolemia como fator de perpetuação da descompensação. Esses achados corroboram o fato de que pacientes com dilatação crônica ventricular desenvolvem mecanismos adaptativos que não permitem ao exame físico perceber a hipervolemia ou a hipovolemia.

Houve queda expressiva da PAD ( $\pm 4 \mathrm{mmHg}$ ) após o protocolo no grupo populacional, porém, esta queda não alcançou significância estatística. Isto, provavelmente, deve-se ao fato de que havia pacientes hipovolêmicos antes do início do protocolo e que, de fato, aumentaram a PVC com a reposição volêmica instituída, contaminando
Tabela IV - Variáveis hemodinâmicas antes e após o protocolo, de todo o grupo

\begin{tabular}{|c|c|c|c|}
\hline Variável & Inicial & Final & Valor de $p$ \\
\hline PAM $(\mathrm{mmHg})$ & $86 \pm 11,91$ & $66 \pm 16,01$ & 0,000838 \\
\hline POAP (mmHg) & $23 \pm 11,50$ & $16 \pm 4,05$ & 0,008975 \\
\hline PVC (mmHg) & $12 \pm 5,88$ & $8 \pm 4,01$ & 0,070158 \\
\hline IRVS (dynes $/ \mathrm{s} / \mathrm{cm}^{-5} / \mathrm{m}^{2}$ ) & $3.023 \pm 1.153,71$ & $1.834 \pm 719,34$ & 0,000155 \\
\hline IC $\left(\mathrm{L} / \mathrm{min} / \mathrm{m}^{2}\right)$ & $2,1 \pm 0,56$ & $2,8 \pm 0,73$ & 0,000386 \\
\hline PAPM (mmHg) & $38 \pm 11,04$ & $28 \pm 7,19$ & 0,006601 \\
\hline
\end{tabular}

Tabela V - Escórias nitrogenadas e eletrólitos antes e após o protocolo

$\begin{array}{lccc}\text { Exame } & \text { Inicial } & \text { Final } & \text { Valor de } p \\ \text { Uréia }(\mathrm{mg} / \mathrm{dL}) & 75,0 \pm 39,2 & 83,7 \pm 47,6 & 0,29 \\ \text { Creatinina }(\mathrm{mg} / \mathrm{dL}) & 1,4 \pm 0,5 & 1,3 \pm 0,5 & 0,70 \\ \text { Sódio }(\mathrm{mEq} / \mathrm{L}) & 135,8 \pm 4,8 & 135,8 \pm 4,6 & 0,83 \\ \text { Potássio }(\mathrm{mEq} / \mathrm{L}) & 4,3 \pm 0,9 & 4,1 \pm 0,4 & 0,61\end{array}$

o resultado global. Quando se analisa a diferença apenas no grupo de hipervolemia, observa-se uma redução ainda mais expressiva, em torno de $5 \mathrm{mmHg}$.

Os resultados deste estudo confirmam que pacientes com IC avançada não só toleram pressões de enchimento reduzidas, como alcançam melhora significativa do índice cardíaco, do índice de volume sistólico e do índice de resistência vascular periférica quando submetidos à $\mathrm{MHI}$. Além disso, apesar de alguns estudos sugerirem que pode haver piora da função renal em pacientes com IC avançada 
submetidos à terapia intensa ${ }^{16,17}$, na população estudada, apesar da intensa diurese obtida e da redução importante da pressão de enchimento ventricular, não se observou piora das escórias. Esses resultados também são semelhantes aos de Rohde e cols. ${ }^{18}$, em que, após intensa redução das pressões de enchimento e da resistência periférica utilizando $\mathrm{MHI}$, foi observado ajuste hemodinâmico sem deterioração da função renal.

Apesar do benefício hemodinâmico observado, como o período de acompanhamento incluiu apenas a fase de internação hospitalar, não foi possível estudar se essa melhora hemodinâmica, e mesmo sintomática, mantém efeito favorável por um período maior de observação.

O benefício hemodinâmico alcançado com as doses de vasodilatadores e diuréticos, por via intravenosa, foi mantido com a transição para as drogas orais nas doses demonstradas. A maioria dos pacientes necessitou de doses plenas de vasodilatadores, por vezes, acima das doses tradicionalmente recomendadas pela literatura. Porém, vale ressaltar que, em alguns casos, doses nãomáximas foram suficientes para ajuste hemodinâmico.

Como o impacto em sobrevida ainda não foi testado com essa estratégia, é fundamental ressaltar que, apesar de em alguns casos ter ocorrido ajuste hemodinâmico com doses menores que as doses plenas dos vasodilatadores, as doses preconizadas nos grandes ensaios clínicos devem sempre ser utilizadas devido ao positivo impacto na sobrevida.

A prática de se usar agentes que aumentam a contratilidade cardíaca (inotrópicos) nos pacientes com $I C$, embora apresente efeitos hemodinâmicos benéficos, está associada ao aumento da mortalidade quando utilizada em longo prazo ${ }^{16}$. Apesar disso, os inotrópicos continuam sendo comumente utilizados no manejo da IC crônica agudizada. A dobutamina foi substituída pelo nitroprussiato de sódio, sem piora hemodinâmica. De fato, houve benefício hemodinâmico adicional ao do inotrópico com a utilização do vasodilatador. Estudo de Capomolla e cols. ${ }^{19}$, comparando o efeito da dobutamina com o do nitroprussiato de sódio em pacientes com IC grave, mostrou que ambos melhoram o débito cardíaco de forma semelhante, porém a dobutamina tem efeito imprevisível na POAP e na regurgitação mitral, enquanto que o nitroprussiato melhora de forma significativa as pressões de enchimento e a regurgitação mitral. Portanto, como houve sucesso em todos os pacientes na retirada da dobutamina e benefício com a estratégia de doses ajustadas de vasodilatadores e diuréticos guiadas pela $\mathrm{MHI}$, o uso de inotrópicos deve ser reservado para aqueles que não respondem a essa estratégia. Abordagem semelhante foi sugerida por Rohde e cols. ${ }^{20}$, na qual uma abordagem escalonada e progressiva deve ser feita, reservando os inotrópicos para aqueles sem benefício com o uso da MHI.

Para responder ao impacto dessa estratégia em relação à mortalidade, recentemente foram apresentados os resultados de um estudo clínico, randomizado, multicêntrico (ESCAPE) ${ }^{21}$, compreendendo 433 pacientes com IC em CF IV, comparando o grupo com estratégia guiada pela $\mathrm{MHI}$ com o outro guiado apenas pelos dados clínicos. Foi observado que a mortalidade ao final de 30 dias, não foi diferente, sendo $4,7 \%$ para o grupo que usou o Swan-Ganz e 5,0\%, para o grupo tratado clinicamente apenas. Os resultados, ao final de seis meses, também não mostraram diferença significativa e estão na tabela VI.

Tabela VI - Objetivos clínicos ao final de 6 meses

\begin{tabular}{|ccc|}
\hline Objetivos & $\begin{array}{c}\text { Swan-Ganz, } \\
n=215(\%)\end{array}$ & $\begin{array}{c}\text { Clinico, } \\
n=218(\%)\end{array}$ \\
\hline Mortalidade & 20,9 & 17,4 \\
\hline Re-hospitalizações/paciente (média) & 2,1 & 2,1 \\
\hline Dias no hospital (média) & 11 & 11 \\
\hline
\end{tabular}

Em relação aos eventos adversos e complicações intrahospitalares, também não houve diferença entre os grupos. Portanto, o estudo mostrou que é seguro utilizar essa estratégia para pacientes com sintomas persistentes de IC e que parece razoável considerar o uso da $\mathrm{MHI}$ para guiar a terapêutica em um paciente individual.

Concluindo, o presente estudo mostrou que é possível, seguro e desejável uma redução importante das pressões de enchimento ventricular, uma vez que essa estratégia implica em melhora significativa do débito cardíaco e da resistência periférica nessa população. Apenas com os dados hemodinâmicos, foi possível identificar pacientes com hipovolemia persistente e outros com hipervolemia não identificada clinicamente e assim adequar o tratamento, com reposição volêmica no primeiro grupo e com uso de doses significativamente maiores de vasodilatadores e diuréticos no outro. Novos métodos de avaliação hemodinâmica não-invasiva devem ser pesquisados, a fim de se tratar um número maior de pacientes com dados objetivos e não apenas com os achados do exame físico.

\section{REFERÊNCIAS}

1. Thomas JT, Kelly RF, Thomas SJ et al. Utility of history, physical examination, electrocardiogram, and chest radiograph for differentiating normal from decreased systolic function in patients with heart failure. Am J Med 2002; 112: 437-45.
2. Vinch CS, Aurigemma GP, Hill JC et al. Usefulness of clinical variables, echocardiography, and levels of brain natriuretic peptide and norepinephrine to distinguish systolic and diastolic causes of acute heart failure. Am J Cardiol 2003; 91: 1140-3. 
3. Stevenson LW, Perloff JK. The limited reliability of physical signs for estimating hemodynamics in chronic heart failure. JAMA $1989 ; 261: 884-8$.

4. CONSENSUS Trial Study Group. Effects of enalapril on mortality in severe congestive heart failure. Results of the Cooperative North Scandinavian Enalapril Survival Study (CONSENSUS). N Engl J Med 1987; 316: 1429-35.

5. Califf RM, Adams KF, McKenna W et al. A randomized controlled trial of epoprostenol therapy for severe congestive heart failure: The Flolan international randomized survival trial (FIRST). Am Heart J 1997; 134: 44-54.

6. Packer M, Coats AJS, Fowler MB et al. Effect of Carvedilol on survival in severe chronic heart failure. N Engl J Med 2001; 344: 1651-8.

7. Boehmer JP. Device therapy for heart failure. Am J Cardiol 2003; 91: 53D-59D.

8. St John Sutton MG, Plappert T, Abraham WT et al. For the Multicenter InSync Randomized Clinical Evaluation (MIRACLE) Study Group. Effect of cardiac resynchronization therapy on left ventricular size and function in chronic heart failure. Circulation 2003; 107: 1985-90.

9. Perin EC, Dohmann HFR, Borojevic R et al. Transendocardial autologous bone marrow cell transplantation for severe, chronic ischemic heart failure. Circulation 2003; 107: r75-r83.

10. Stevenson LW, Tillisch JH, Hamilton M et al. Importance of hemodynamic response to therapy in predicting survival with ejection fraction $\leq 20 \%$ secondary to ischemic or nonischemic cardiomyopathy. Am J Cardiol 1990;66:1348-54.

11. Mehra A, Ostrzega E, Shotan A, Johnson JV, Elkayam U. Persistent hemodynamic improvement with short-term nitrate therapy in patients with chronic congestive heart failure already treated with captopril. Am J Cardiol 1992;70:1310-14.
12. Lucas C, Johnson W, Hamilton MA et al. Freedom from congestion predicts good survival despite previous class IV symptoms of heart failure. Am Heart J 2000;140:840-7.

13. Stevenson LW, Dracup KA, Tillisch JH. Efficacy of medical therapy tailored for severe congestive heart failure in patients transferred for urgent cardiac transplantation. Am J Cardiol 1989;63:461-4.

14. Packer M, Carver JR, Rodeheffer RJ et al. Effect of oral milrinone on mortality in severe chronic heart failure. The Promise Study Research Group. N Engl J Med 1991;325:1468-75.

15. Cohn JN, Goldstein SO, Greenberg BH et al. A dose-dependent Increase in mortality with vesnarinone among patients with severe heart failure. N Engl J Med 1998;339:1810-6.

16. O'Connor CM, Gattis WA, Uretsky BF et al. Continuous intravenous dobutamine is associated with an increased risk of death in patients with advanced heart failure: insights from the Flolan International Randomized Survival Trial (FIRST). Am Heart J 1999;138:78-86.

17. Weinfeld MS, Chertow GM, Stevenson LW. Aggravated renal dysfunction during intensive therapy for advanced chronic heart failure. Am Heart J 1999;138:285-90.

18. Rohde LE, Furian T, Campos $C$ et al. Implications of the hemodynamic optimization approach guided by right heart catheterization in patients with severe heart failure. Arq Bras Cardiol 2002;78:261-6.

19. Capomolla S, Pozzoli M, Opasich C et al. Dobutamine and nitroprusside infusion in patients with severe congestive heart failure: hemodynamic improvement by discordant effects on mitral regurgitation, left atrial function, and ventricular function. Am Heart J 1999;134:1089-98.

20. Rohde LE, Polanczyk CA, Ribeiro JP, Clausell N. Otimização das indicações e do manejo terapêutico de pacientes referidos para transplante cardíaco. Arq Bras Cardiol 1996;67:189-93.

21. Shah MR, O'Connor CM, Sopko G, Hasselblad V, Califf RM, Stevenson LW. Evaluation Study of Congestive Heart Failure and Pulmonary Artery Catheterization Effectiveness (ESCAPE): design and rationale. Am Heart J 2001; 141: 528-35. 